\title{
Strongest Ferromagnetic Coupling in Designed Gadolinium(III)-Nitroxide Coordination Compounds
}

\author{
Takuya Kanetomo, Toru Yoshitake, and Takayuki Ishida* \\ Department of Engineering Science, The University of Electro-Communications, \\ Chofu, Tokyo 182-8585, Japan. \\ E-mail: takayuki.ishida@uec.ac.jp
}

Supporting Information 
a)

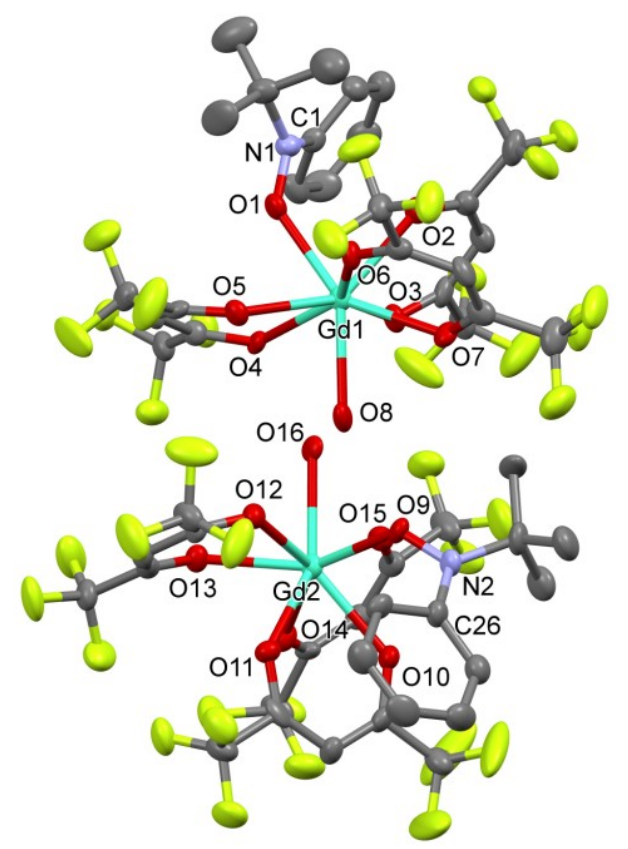

b)

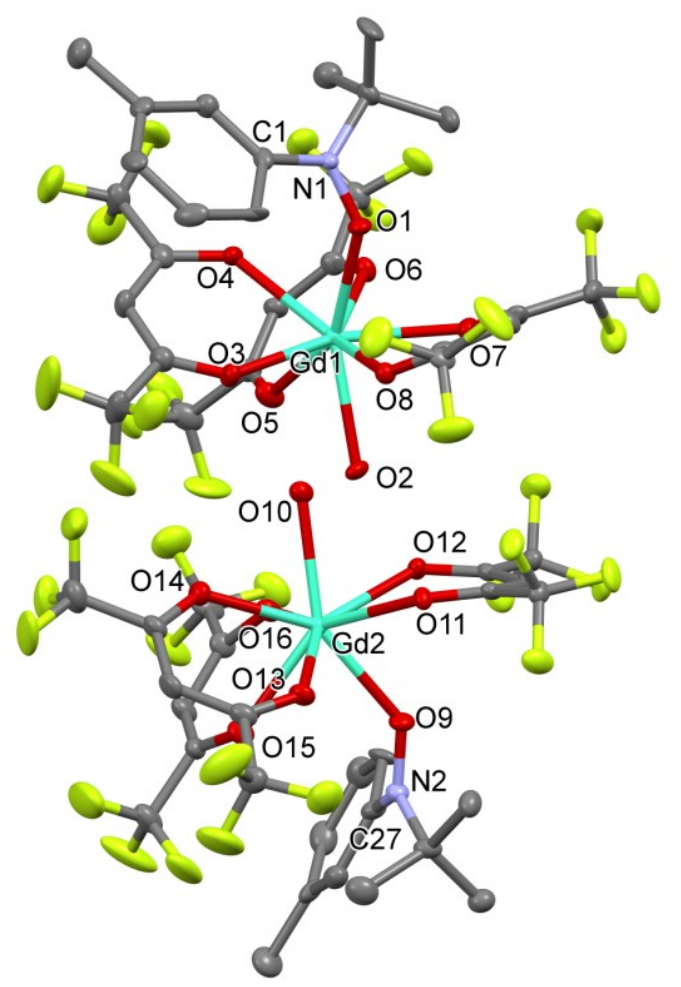

c)

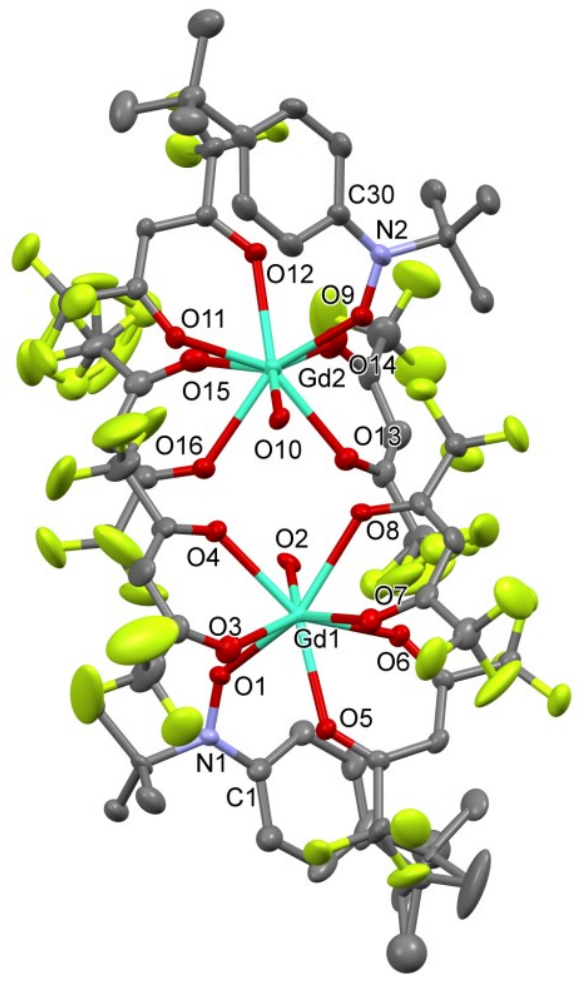

Figure S1. X-ray crystal structures of (a) Gd-phNO, (b) Gd-3tolNO, and (c) Gd-4tbphNO with thermal ellipsoids at the $50 \%$ level. Hydrogen atoms are omitted for clarity. 
Table S1. Coordination polyhedra, exchange parameters, and dihedral angles of Gd-radical compounds.

\begin{tabular}{|c|c|c|c|c|c|c|c|c|c|}
\hline \multirow{3}{*}{$\begin{array}{l}\text { Compound } \\
{\left[\mathrm{Gd}(\mathrm{hfac})_{3}(\mathrm{phNO})\left(\mathrm{H}_{2} \mathrm{O}\right)\right]^{\mathrm{a}, \mathrm{b}}}\end{array}$} & \multirow{3}{*}{$\begin{array}{l}2 J / k_{\mathrm{B}} \mathrm{K} \\
+18.0\end{array}$} & \multirow{3}{*}{$\begin{array}{l}|\phi \phi|^{\circ} \\
70.3 \\
69.2\end{array}$} & \multicolumn{6}{|c|}{ Shape measures ${ }^{\mathrm{y}}$ (the 1st, 2nd, and 3rd solutions) } & \multirow{3}{*}{$\begin{array}{c}\text { Ref. } \\
\text { this work }\end{array}$} \\
\hline & & & TDD & 0.820 & SAPR & 1.409 & BTPR & 1.490 & \\
\hline & & & SAPR & 1.268 & BTPR & 1.353 & TDD & 1.835 & \\
\hline \multirow{2}{*}[\mathrm{Gd}(\mathrm{hfac})_{3}(3\mathrm{tolNO})(\mathrm{H}_{2}\mathrm{O})]{$^{\mathrm{a}, \mathrm{c}}$} & \multirow{2}{*}{+16.7} & 72.3 & BTPR & 1.025 & SAPR & 1.394 & TDD & 1.514 & \multirow{2}{*}{ this work } \\
\hline & & 77.8 & BTPR & 0.989 & SAPR & 1.433 & TDD & 1.840 & \\
\hline$\left[\mathrm{Gd}(\mathrm{hfac})_{3}(\mathrm{NITPh}-3-\mathrm{Br}-4 \mathrm{OMe})_{2}\right]^{\mathrm{a}, \mathrm{d}}$ & +16.4 & 79.96 & TDD & 0.340 & SAPR & 1.718 & BTPR & 2.416 & 1 \\
\hline$\left[\mathrm{Gd}\left(\mathrm{NO}_{3}\right)_{3}(\text { nittrz })_{2}\right]^{\mathrm{e}}$ & +8.77 & 53.8 & JBCSAPR & 3.223 & JSPC & 3.511 & TD & 6.295 & 2 \\
\hline \multirow{2}{*}[\mathrm{Gd}(\mathrm{hfac})_{3}(4\mathrm{tbphNO})(\mathrm{H}_{2}\mathrm{O})]{$^{\mathrm{a}, \mathrm{f}}$} & \multirow{2}{*}{+7.5} & 58.2 & SAPR & 0.737 & TDD & 1.034 & BTPR & 1.416 & \multirow{2}{*}{ this work } \\
\hline & & 61.3 & SAPR & 0.552 & TDD & 1.527 & BTPR & 1.688 & \\
\hline$\left[\mathrm{Gd}(\mathrm{hfac})_{3}(\mathrm{NIT}-5-\mathrm{Br}-3 \mathrm{py})\right]_{2}{ }_{2}^{\mathrm{a}, \mathrm{g}}$ & +7.44 & 93.85 & TDD & 0.301 & BTPR & 2.598 & JSD & 2.761 & 3 \\
\hline$\left[\mathrm{Gd}(\mathrm{hfac})_{3}(\mathrm{NITPhiPr})_{2}\right]^{\mathrm{a}, \mathrm{h}}$ & +6.92 & 84.6 & TDD & 0.159 & SAPR & 1.965 & BTPR & 2.312 & 4 \\
\hline$\left[\mathrm{Gd}(\mathrm{hfac})_{3} \mathrm{~L}_{2}\right]^{\mathrm{a}, \mathrm{i}}$ & +6.9 & 87.6 & TDD & 0.285 & SAPR & 2.081 & BTPR & 2.678 & 5 \\
\hline$\left[\mathrm{Gd}(\mathrm{hfac})_{3}\left(\mathrm{NITPhPO}(\mathrm{OEt})_{2}\right)\right]_{2}{ }^{\mathrm{a}, \mathrm{j}}$ & +4.98 & 90.11 & TDD & 0.714 & SAPR & 1.295 & BTPR & 1.656 & 6 \\
\hline$\left[\mathrm{Gd}(\mathrm{hfac})_{3}(\mathrm{NITBz} \operatorname{ImH})\right]^{\mathrm{a}, \mathrm{k}}$ & +4.89 & 38.3 & SAPR & 0.434 & TDD & 1.762 & BTPR & 1.967 & 7 \\
\hline$\left[\mathrm{Gd}(\mathrm{hfac})_{3}(\mathrm{NIToPy})\right] \cdot 0.5 \mathrm{C}_{7} \mathrm{H}_{16}{ }^{\mathrm{a}, \mathrm{l}}$ & +4.36 & 50.1 & SAPR & 1.003 & TDD & 1.407 & BTPR & 1.534 & 8 \\
\hline$\left[\mathrm{Gd}(\mathrm{hfac})_{3}\left(\mathrm{NITPhOCH}_{3}\right)_{2}\right]^{\mathrm{a}, \mathrm{m}}$ & +4.26 & 83.32 & TDD & 0.219 & SAPR & 1.776 & BTPR & 1.991 & 9 \\
\hline$\left[\mathrm{Gd}(\mathrm{hfac})_{3}\left(\mathrm{NITPh}\left(\mathrm{OCH}_{3}\right)_{3}\right)\right]_{2}{ }^{\mathrm{a}, \mathrm{n}}$ & +4.20 & 91.92 & TDD & 0.238 & SAPR & 1.917 & BTPR & 2.263 & 10 \\
\hline$\left[\mathrm{Gd}(\mathrm{hfac})_{3}(\mathrm{HNN})_{3}\right]^{\mathrm{a}, \mathrm{o}}$ & +3.86 & 42.6 & TCTPR & 0.460 & CSAPR & 0.480 & MFF & 0.927 & 11 \\
\hline$\left[\mathrm{Gd}(\mathrm{hfac})_{3}(\mathrm{HNN})_{2}\right]^{\mathrm{a}, \mathrm{o}}$ & +2.61 & 45.2 & SAPR & 0.253 & BTPR & 2.115 & TDD & 2.330 & 12 \\
\hline$\left[\mathrm{Gd}(\mathrm{hfac})_{3}(\mathrm{NITPh})_{2}\right]^{\mathrm{a}, \mathrm{p}}$ & +1.77 & 88.07 & TDD & 0.177 & BTPR & 1.889 & SAPR & 1.969 & 13 \\
\hline$\left[\mathrm{Gd}(\mathrm{hfac})_{3}\left(\mathrm{NITPhOCH}_{2} \mathrm{Ph}\right)_{2}\right]^{\mathrm{a}, \mathrm{q}}$ & +1.77 & 81 & TDD & 0.452 & SAPR & 1.131 & BTPR & 1.869 & 14 \\
\hline$\left[\mathrm{Gd}(\mathrm{hfac})_{3}(\mathrm{NITPhOEt})_{2}\right]^{\mathrm{a}, \mathrm{r}}$ & +0.772 & 82.95 & TDD & 0.270 & SAPR & 1.605 & BTPR & 1.832 & 14 \\
\hline$\left[\mathrm{Gd}(\mathrm{hfac})_{3}\left(\mathrm{NITPhOC}_{4} \mathrm{H}_{9}\right)_{2}\right]^{\mathrm{a}, \mathrm{s}}$ & +0.273 & 83.57 & TDD & 0.262 & SAPR & 1.643 & BTPR & 1.828 & 15 \\
\hline$\left[\mathrm{Gd}\left(\mathrm{NO}_{3}\right)_{3}(\mathrm{NITBz} \operatorname{ImH})_{2}\right]^{\mathrm{k}}$ & -6.93 & 36.0 & JSPC & 3.043 & TD & 3.484 & JBSAPR & 3.910 & 16 \\
\hline$\left[\mathrm{Gd}(\mathrm{hfac})_{3}(\mathrm{IMBz} \operatorname{ImH})\right]^{\mathrm{a}, \mathrm{t}}$ & -7.4 & 5.59 & SAPR & 0.633 & TDD & 1.759 & BTPR & 1.825 & 7 \\
\hline$\left[\mathrm{Gd}(\mathrm{hfac})_{3}(\mathrm{IM}-2 \mathrm{py})\right]^{\mathrm{a}, \mathrm{u}}$ & -8.628 & 17.53 & TDD & 0.791 & BTPR & 1.561 & SAPR & 1.937 & 27 \\
\hline$\left[\mathrm{Gd}(\mathrm{hfac})_{3}(2 \mathrm{pyNO})\left(\mathrm{H}_{2} \mathrm{O}\right)\right]^{\mathrm{a}, \mathrm{v}}$ & -13.8 & 19.5 & CSAPR & 0.715 & MFF & 1.010 & TCTPR & 1.085 & 12 \\
\hline$\left[\mathrm{Gd}(\mathrm{hfac})_{3}(6 \mathrm{bpyNO})\right]^{\mathrm{a}, \mathrm{w}}$ & -15.9 & 16.6 & CSAPR & 0.258 & MFF & 1.000 & TCTPR & 1.086 & 18 \\
\hline$\left[\mathrm{Gd}\left(\mathrm{Hbpz}_{3}\right)_{2}(\mathrm{dtbsq})\right] \cdot 2 \mathrm{CHCl}_{3}{ }^{\mathrm{x}}$ & -16.4 & 4.73 & SAPR & 1.060 & TDD & 1.169 & BTPR & 1.978 & 19 \\
\hline$\left[\mathrm{K}\left(18\right.\right.$-crown-6)] $\left\{\left[\left(\mathrm{Me}_{3} \mathrm{Si}\right)_{2} \mathrm{~N}\right]_{2}(\mathrm{THF}) \mathrm{Gd}\right\}_{2}$ & -38.8 & 0 & SPY & 7.831 & TBPY & 11.107 & vOC & 12.361 & 20 \\
\hline
\end{tabular}

${ }^{\mathrm{a}} \mathrm{Hhfac}=1,1,1,5,5,5$-hexafluoropentane-2,4-dione. ${ }^{\mathrm{b}} \mathrm{phNO}=t$-butyl phenyl nitroxide. ${ }^{\mathrm{c}} 3$ tolNO $=t$-butyl 3-tolyl nitroxide. ${ }^{\mathrm{d}} \mathrm{NITPh}-3 \mathrm{Br}-4 \mathrm{OMe}$ = 2-(3-bromo-4-methoxyphenyl)-4,4,5,5-tetramethylimidazolin-1-oxyl 3-oxide. ${ }^{\mathrm{e}}$ nittrz $=2$-(4,5-dimethyl-4H-1,2,4-triazole-3-yl)-4,4,5,5tetramethylimidazolin-1-oxyl 3-oxide. ${ }^{\mathrm{f}} 4 \mathrm{tbphNO}=t$-butyl 4-t-butylphenyl nitroxide. ${ }^{\mathrm{g}} \mathrm{NIT}-5$-Br-3py $=2$-(5-bromo-3-pyridyl)-4,4,5,5tetramethylimidazolin-1-oxyl 3-oxide. ${ }^{\mathrm{h}} \mathrm{NITPhiPr}=2$-(4-isopropylphenyl)-4,4,5,5-tetramethylimidazolin-1-oxyl 3-oxide. ${ }^{\mathrm{i}} \mathrm{L}=2$-[1-(4methylquinolin-2-yl)-1 $H$-pyrazol-4-yl]- 4,4,5,5-tetramethylimidazolin-1-oxyl 3-oxide. ${ }^{j} \mathrm{NITPhPO}(\mathrm{OEt})_{2}=2$-(4-diethoxyphosphorylphenyl)4,4,5,5-tetramethylimidazolin-1-oxyl 3-oxide. ${ }^{k}$ NITBzImH $=2$-(2-benzimidazolyl)-4,4,5,5-tetramethylimidazolin-1-oxyl 3-oxide. ${ }^{1}$ NIToPy $=$ 2-(2-pyridyl)-4,4,5,5-tetramethylimidazolin-1-oxyl 3-oxide. ${ }^{\mathrm{m}} \mathrm{NITPhOCH}_{3}=$ 2-(4-methoxyphenyl)-4,4,5,5-tetramethylimidaozlin-1-oxyl 3-oxide. ${ }^{\mathrm{n}} \mathrm{NITPh}\left(\mathrm{OCH}_{3}\right)_{3}=2$-(3,4,5-trimethoxyphenyl)-4,4,5,5-tetramethylimidazolin-1-oxyl 3-oxide ${ }^{\circ} \mathrm{HNN}=4,4,5,5$-tetramethylimidazolin-1-oxyl 3-oxide. ${ }^{\mathrm{p}} \mathrm{NITPh}=2$-phenyl-4,4,5,5-tetramethylimidazolin-1-oxyl 3-oxide. ${ }^{\mathrm{q}} \mathrm{NITPhOCH}_{2} \mathrm{Ph}=2$-(4-benzyloxyphenyl)-4,4,5,5tetramethylimidazolin-1-oxyl 3-oxide. ${ }^{\mathrm{r}} \mathrm{NITPhOEt}=2$-(4-ethoxyphenyl)-4,4,5,5-tetramethylimidazolin-1-oxyl 3-oxide. ${ }^{\mathrm{s}} \mathrm{NITPhOC}_{4} \mathrm{H}_{9}=$ 
2-(4-butoxyphenyl)-4,4,5,5-tetramethylimidazolin-1-oxyl 3-oxide. ${ }^{\mathrm{t}} \mathrm{IMBzImH}=2$-(2-benzimidazolyl)-4,4,5,5-tetramethylimidazolin-1-oxyl.

${ }^{\mathrm{u}} \mathrm{IM}-2$ py $=2$-(2-pyridyl)-4,4,5,5-tetramethylimidazolin-1-oxyl. ${ }^{\mathrm{v}} 2$ pyNO $=t$-butyl 2-pyridyl nitroxide. ${ }^{\mathrm{w}} 6 \mathrm{bpyNO}=2,2$ '-bipyridin-6-yl $t$-butyl nitroxide. ${ }^{\mathrm{x}} \mathrm{Hbpz}_{3}=$ hydrotris(pyrazolyl)borate, $\mathrm{dtbsq}=3,5$-di-t-butylsemiquinonate.

${ }^{\mathrm{y}}$ Five-coordination polyhedra: SPY $=$ square pyramid. TBPY $=$ trigonal bipyramid. vOC $=$ vacant octahedron. Eight-coordination polyhedra: $\mathrm{TDD}=$ triangular dodecahedron. $\mathrm{SAPR}=$ square antiprism. BTPR $=$ biaugmented trigonal prism. JSD $=$ snub disphenoid. Nine-coordination polyhedra: $\mathrm{TCTPR}=$ tricapped trigonal prism. $\mathrm{CSAPR}=$ capped square antiprism. $\mathrm{MFF}=$ muffin. Ten-coordination polyhedra: $\mathrm{JBCSAPR}=$ bicapped square antiprism. JSPC $=$ sphenocorona. TD $=$ tetradecahedron.

\section{References}

1 Wang, Y. -L.; Gao, Y. -Y.; Ma, Y.; Wang, Q. -L.; Li, L. -C.; Liao, D. -Z. J. Solid State Chem. 2013, $202,276$.

2 Sutter, J. -P.; Kahn, M. L.; Golhen, S.; Ouahab, L.; Kahn, O. Chem. Eur. J. 1998, 4, 571.

3 Xu, J.-X.; Ma, Y.; Xu, G.-F.; Wang, C.; Liao, D.-Z.; Jiang, Z.-H.; Yan, S.-P.; Li, L.-C. Inorg. Chem. Commun. 2008, 11, 1356.

4 Gao, Y. -y.; Wang, Y. -1.; Hu, P.; Yang, M. -f.; Ma, Y.; W. Q. -1.; Li, L. -c.; Liao, D. -z. Inorg. Chem. Commun. 2013, 27, 31.

5 Tretyakov, E. V.; Fokin, S. V.; Zueva, E. M.; Tkacheva, A. O.; Romanenko, G. V.; Bogomyakov, A. S.; Larionov, S. V.; Popov, S. A.; Ovcharenko, V. I. Russ. Chem. Bull., Int. Ed. 2014, 63, 1.

6 Pointillart, F.; Bernot, K.; Poneti, G.; Sessoli, R. Inorg. Chem. 2012, 51, 12218.

7 Lescop, C.; Luneau, D.; Rey, P.; Bussière, G.; Reber, C. Inorg. Chem. 2002, 41, 5566.

8 Benelli, C.; Caneschi, A.; Gatteschi, D.; Pardi, D. Inorg. Chem. 1992, 31, 741.

9 Zhao, Q. -H.; Ma, Y. -P.; Du, L.; Fang, R. -B. Trans. Met. Chem. 2006, 31, 593.

10 Zhang, C. -X.; Sun, N. -N.; Zhao, X. -Y.; Zhang, Y. -Y.; Guo, Y. -L. Inorg. Chem. Commun. 2011, $14,166$.

11 Ikegaya, N.; Kanetomo, T.; Murakami, R.; Ishida, T. Chem. Lett. 2012, 41, 82.

12 Ishida, T.; Murakami, R.; Kanetomo, T.; Nojiri, H. Polyhedron 2013, 66, 183.

13 Benelli, C.; Caneschi, A.; Gatteschi, D.; Pardi, L.; Rey, P.; Shum, D. P.; Carlin, R. L. Inorg. Chem. 1989, 28, 272.

14 Zhou, N.; Ma, Y.; Wang, C.; Xu, G.-F.; Tang, J.; Yan, S.-P.; Liao, D.-Z. J. Solid State Chem. 2010, $183,927$. 
15 Wang, X. -L. Inorg. Chim. Acta 2012, 387, 20.

16 Lescop, C.; Belorizky, E.; Luneau, D.; Ray, P. Inorg. Chem. 2002, 41, 3375.

17 Tsukuda, T.; Suzuki, T.; Kaizaki, S. J. Chem. Soc., Dalton Trans. 2002, 1721.

18 Kanetomo, T.; Ishida, T. Inorg. Chem. 2014, 53, 10794.

19 Caneschi, A.; Dei, A.; Gatteschi, D.; Sorace, L.; Vostrikova, K. Angew. Chem. Int. Ed. 2000, 39, 246.

20 Rinehart, J. D.; Fang, M.; Evans, W. J.; Long, J. R. Nat. Chem. 2011, 3, 538. 